\title{
ELABORACIÓN DE UN PERFIL OCUPACIONAL PARA UNA ADECUADA UBICACIÓN DEL JOVEN CON RETARDO MENTAL EN UN TALLER OCUPACIONAL
}

\section{Amparo Sotil Brioso ${ }^{1}$}

La investigación establece los perfiles ocupacionales del educando con retardo mental considerando sus reales potencialidades, habilidades, destrezas y aptitudes, contribuyendo así a una adecuada ubicación en un Taller Ocupacional. mejorando su preparación, y de esta manera hacer factible su inclusión en el mundo laboral..

Palabras Clave: Educación ocupacional, perfil ocupacional, inserción laboral.

The research establishes the occupational profiles of the student with mental retardation considering their real potentialities; abilities, skills and aptitudes, contributing in that way to an adequate allocation in a Occupational Workshop improving their preparation, and by this way making possible their inclusion in the labor world.

Key Words: Occupational education, occupational profile, labor inclusion.

1 Miembros de la investigación: Emma Campos Pacheco y Graciela Noriega Reynoso. 
La importancia de establecer el Perfil Profesional del Educando que presenta retardo mental, contribuyendo de esta manera a que en el tiempo más corto, se ubiquen en el taller en que posteriormente se van a perfeccionar, y no colocándolos al azar, ya que es el método menos indicado en estos casos especiales, por cuanto no sólo se afecta el aspecto académico, sino también se intensifican los problemas en el área de personalidad, pues los niños se frustran al no obtener logros, incrementándose la angustia, ansiedad, inseguridad emocional, baja autoestima, etc., que por su problemática desde ya la tienen afectada.

Este instrumento también contribuye a una mejor preparación y de esta manera a hacer factible la inclusión del deficiente mental en el mundo laboral.

\section{FORMULACIÓN DEL PROBLEMA}

¿En qué medida se tiene en cuenta las habilidades, capacidades e intereses de los alumnos con retardo mental que están ubicados en los diferentes talleres de ocupación laboral como son: carpintería, costura, zapatería, encuadernación, jardinería-cosmetología, juguetería, cerámica y cotillón?

\section{OBJETIVOS}

1. Establecer el Perfil Ocupacional del educando que presenta retardo mental, considerando su capacidad real así como sus habilidades y destrezas.

2. Determinar las áreas altas y bajas del educando con retardo mental en relación a los aspectos: exactitud, agilidad y armonía, factores determinantes para establecer la edad motriz, la que constituye un factor importante para su rendimiento en los talleres ocupacionales.

3. Determinar el grado de socialización y ocupación del educando con retardo mental. 


\section{IMPORTANCIA DEL ESTUDIO}

La formación laboral constituye uno de los aspectos básicos dentro del Programa global de atención al deficiente mental. Uno de los aspectos que nos preocupan más es la dificultad para su ubicación en el mundo del trabajo. Comprendemos que esta exclusión tiene su origen en las propias limitaciones del sujeto y en la características de nuestro medio laboral, pero comprendemos así mismo, que si bien los Centros de Educación Especial en el Perú han sido creados para integrar al deficiente mental en el campo laboral, sin embargo, al observar en detalle la estructura curricular, nos damos cuenta que existen desfases que dificultan el cumplimiento de este objetivo. El gobierno en su proyecto de Ley de Bases de la Persona Impedida, dado por el Congreso el 16 de Octubre de 1995 por los congresistas: Javier Diez Canseco, Roger Guerra García, Graciela Fernández Baca, Ernesto Gamarra, Gustavo Mohme y Carlos Ferrero Costa, en el capitulo VI (De la Educación), articulo 18, inciso c, señala: "Los Centros de Educación Especial y los Programas integrados acentuarán en sus planes de acción la orientación vocacional y la capacitación para el trabajo".

En el Capítulo VII (Del Trabajo), artículo 21, señala lo siguiente:

"Declárese de interés social la protección, atención y readaptación laboral del impedido a fin de permitirle acceder y/o conservar y/o crear un empleo adecuado y progresar en el mismo, favoreciendo su acceso a la capacitación y al crédito".

Considerando que el Estado apoya al impedido obligando al sector y a las empresas privadas a darles empleo, es necesario perfeccionar el currículum de estudios vigente para que de esta manera responda a las exigencias solicitadas por las empresas públicas o privadas. Por ello nos proponemos dar aportes con la elaboración de un perfil ocupacional que permita lograr una mejor ubicación en un Taller Ocupacional. 


\section{ANÁLISIS OCUPACIONAL *}

La aplicación de las técnicas de análisis (Dos Santos, 1977) permite obtener una serie de resultados directos que constituyen los productos del proceso de análisis ocupacional.

Los productos del análisis ocupacional, en una aplicación concreta dependerán de las necesidades específicas que, en materia de información sobre las ocupaciones, plantee el proceso en el que van a ser utilizados, los datos ocupacionales. Estos procesos, que emplean los datos resultantes del análisis como información de entrada, constituyen las aplicaciones del análisis ocupacional.

Los principales productos del análisis ocupacional desde el punto de vista de sus aplicaciones en los campos de la formación ocupacional y la certificación ocupacional, son los siguientes:

- Definición de talleres ocupacionales.

- Descripción del trabajo ejecutado en las ocupaciones.

- Condiciones ambientales.

- Demandas físicas.

- Exigencias de las ocupaciones.

- Inventarios de ocupaciones.

- Niveles de calificación de las ocupaciones.

- Perfiles ocupacionales.

- Clasificación de las ocupaciones.

- Desarrollo educacional general. Educación general que se requiere para el ejercicio de una ocupación y que se obtiene por medio de la enseñanza básica y especialización.

- Aptitudes. Capacidades específicas requeridas de una persona para aprender o ejecutar debidamente las tareas de la ocupación.

* INACAP. Op. Cit., pp. 5 Y 6.- SENA. División de Metodología y Formación. Análisis ocupacional y análisis del puesto de trabajo. Bogotá, 1976, p. 8, Unidad Autoformativa, n. 5.- Joao G. Dos Santos, Educaodo e analise ocupacional. Rio de Janeiro, SENAI, Demarcación Nacional, 1977, v. 1. pp. 3 Y 4. 
- Intereses. Preferencias por ciertos tipos de actividades o experiencias

que se acompañan de un rechazo a las actividades o experiencias contrarías a ellas.

Temperamentos: Diferentes tipos de situaciones ocupacionales a las cuales los trabajadores deben ajustarse.

En el presente trabajo serán tomados en cuenta los factores enunciados.

\section{METODOLOGÍA}

\section{Tipo y diseño de investigación}

La presente investigación es de tipo descriptivo comparativo y el diseño empleado es pre-experimental.

\section{Variables}

V.I. : Aptitudes, destrezas y características de personalidad de los educandos con retardo mental.

V.D. : Rendimiento en los diferentes talleres.

V.C. : Grado de retardo: leve y moderado.

Talleres Ocupacionales: carpintería, bio-huerto, costura, decoraciones, cosmetología, cerámica.

\section{Muestra}

La muestra estuvo conformada por 110 jóvenes de ambos sexos que cursan estudios en talleres ocupacionales de Centros de Educación Especial a nivel Estatal en Lima.

\section{Técnicas e Instrumentos}

Técnicas utilizadas:

a) Observación de los alumnos en el aula, los métodos que se 
utilizan para ubicarlos, grados de adaptación y nivel de ejecución con el fin de seleccionar el taller en el que se deben ubicar.

b) Capacitación a profesores y padres de familia a través del Programa de Escuela para Padres, para una mejor aceptación y comprensión del sistema de rotación de los alumnos por los diferentes Talleres del Centro Educativo.

A los profesores se les capacitó sobre técnicas que se emplearían para la elaboración del perfil a fin de mejorar su nivel de información y fortalecer el trabajo en equipo.

Instrumentos utilizados:

Test del examen de la coordinación visomotriz y dinámica manual de Dalila Molina de Costallat (PAC). Esta prueba evalúa exactitud, agilidad y armonía de movimientos con el fin de determinar las edades motrices desde los 5 hasta los 10 años.

a) Socialización: Se trata aquí aquellas habilidades y conductas que permiten una convivencia armónica estableciendo y manteniendo relaciones con los que le rodean, facilitando el intercambio de experiencias y el enriquecimiento personal a través de los beneficios que la vida en comunidad le proporciona.

b) Ocupación: Esta área comprende las destrezas y habilidades necesarias del individuo para incorporarse en forma efectiva a la sociedad.

\section{PROCESAMIENTO Y ANÁLISIS DE DATOS}

Los datos han sido organizados según las variables establecidas por el diseño de investigación y tratados en base a los siguientes estadísticos:

a) Distribución porcentual de los niveles diagnósticos alcanzados en las áreas de socialización y ocupación del PAC.

b) Distribución porcentual de la edad motriz de acuerdo a cada uno 
de los factores considerados en la prueba: exactitud, agilidad y armonía.

c) Prueba $\mathrm{Z}$ de las edades medias alcanzadas en los diversos Talleres ocupacionales en las habilidades motoras básicas para cada uno de los tres factores considerados: armonía, exactitud y agilidad.

d) Análisis de varianza para las diferencias de medidas en la edad motriz en sus tres factores: exactitud, agilidad y armonía.

e) Evaluación de perfiles para cada uno de los siguientes talleres: carpintería, costura, cerámica, cosmetología, biohuerto y decoración.

\section{RESULTADOS}

En cuanto a la evaluación de la coordinación motriz la dinámica manual a través de la prueba de habilidades motoras básicas de Dalila Molina de Gostallat encontramos los siguientes hallazgos:

a) En lo referente al factor de precisión, el $62.16 \%$ de los examinados se ubica en la edad motriz correspondiente a 8 años. El $20.27 \%$ se ubica en la edad motriz correspondiente a 7 años. El $13.5 \%$ se ubica en la edad motriz de 9 años y el $1.35 \%$ se ubica en la edad motriz de 10 años.

b) En lo que concierne al factor de agilidad, los examinados alcanzan la siguiente edad motriz: el $45.94 \%$ ubicándose en 8 años. El 37.83\% ubicándose en 7 años. El 12.16\% se ubica en 9 años y el $4.05 \%$ se ubica en 10 años.

c) Respecto al factor de armonía, encontramos que los examinados se ubican en la siguiente edad motriz: el 54.79\% en 8 años, mientras que el $34.24 \% 7$ años. El $8.21 \% 5$ años y el $1.36 \% 9$ años y en igual porcentaje en la edad motriz de 10 años.

d) En cuanto a los niveles de socialización y ocupación evaluados a través del Inventario de Desarrollo Social de Gunzburg PAC 
forma 2 encontramos que en el área de socialización el $81.8 \%$ se ubica en el nivel diagnóstico medio, mientras que el $13.51 \%$ se ubica en el nivel diagnóstico alto. El $2.7 \%$ se ubica en el nivel diagnóstico excelente y el mismo porcentaje de examinados se encuentra en el nivel diagnóstico inferior.

e) En lo que respecta al área de ocupación, los hallazgos encontrados indican que el $56.75 \%$ se ubica en el nivel diagnóstico medio, mientras que el $32.43 \%$ se ubica en el nivel diagnóstico excelente, por otro lado, el $8.1 \%$ en el nivel diagnóstico inferior.

Los resultados obtenidos se deben a la eficiencia del programa de capacitación laboral aplicado.

a) En base a la prueba $\mathrm{Z}$ de diferencia de medias encontramos diferencias significativas a un nivel de significación del 0.05 en función a los talleres ocupacionales de: decoración, cosmetología, costura, carpintería, biohuerto y cerámica, en los tres factores motrices: exactitud, agilidad y armonía, lo cual demuestra que es importante tener en cuenta el perfil del alumno en relación a los requerimientos de cada especialidad.

b) El análisis de varianza nos ha permitido establecer que las diferencias de los promedios de la edad motriz en sus tres factores: exactitud, agilidad y armonía no difieren significativamente. Lo cual confirma la validez de estos factores en la determinación de la edad motriz.

c) En lo concerniente a la socialización encontramos que gracias al programa de capacitación continua con los profesores y padres de familia se observa un incremento en los niveles alcanzados en la muestra, el mismo que se ubica en el nivel promedio, por cuanto en una etapa inicial el mayor número estaba ubicado en la categoría inferior. 
d) En el área de ocupación el mayor porcentaje de la población se concentra en el nivel promedio, al nivel de diagnóstico medio, lo que nos indica que debemos trabajar en las áreas de motricidad fina para obtener un mayor éxito.

\section{CONCLUSIONES Y RECOMENDACIONES}

1. Se puede afirmar que la edad motriz, el grado de socialización y el desempeño ocupacional son buenos predictores de un rendimiento adecuado en el Área de Rehabilitación Profesional. El análisis ocupacional teniendo en cuenta los factores motrices así como de adaptación nos ha permitido enfocar el perfil centrado en el potencial de ejecución del alumno, el mismo que es valioso para los Centros de Educación Especial que cuentan con Talleres de Rehabilitación Profesional.

2. Los perfiles establecidos para cada especialidad permitirán que los educandos sean ubicados de acuerdo a su real potencial, con mayor rapidez, precisión y objetividad, ya la vez, reducen el tiempo de evaluación, lo cual era un factor limitante para lograr su entrenamiento correspondiente, se tendrá en cuenta los siguientes aspectos:

- Exactitud: Paciencia y pulso

- Agilidad : Destreza manual

Destreza digital

- Armonía : Coordinación bimanual

- Socialización y desempeño ocupacional

- Atención - Concentración - Coordinación visomotriz -Discriminación táctil - Control de impulso - Tolerancia a la frustración Discriminación de lo fundamental de lo accesorio con el fin de seleccionar el taller en el que se les deben ubicar.

3. Considerando que sólo el $1.35 \%$ de los educandos evaluados que actualmente vienen cursando estudios en los Talleres Ocupacionales alcanzan la edad motriz de 10 años, considero pertinente se refuerce e intensifiquen las acciones de estimulación temprana y aprestamiento laboral, en los niveles de Educación Inicial y Primaria Especial, para lo cual es necesario reformular la estructura curricular vigente, que actualmente privilegia las áreas cognitiva y valorativa. De esta forma al adquirir el alumno un mayor repertorio de habilidades y destreza 
motora fina, mediante una programación pre-vocacional, estarían en condiciones de tener un mejor desempeño laboral, cuando tengan que adaptarse y ajustar su rendimiento a las exigencias de un Taller Ocupacional.

4. Observamos asimismo escaso repertorio de habilidades sociales por parte de la población muestral, y siendo el factor social importante para su futura incorporación a su Centro Laboral Estatal o Privada donde el egresado debe poner buena capacidad para relacionarse positivamente con miembros de su entorno y practicar normas de urbanidad y respeto, acatamiento de reglamentos y disciplina laboral, así como de horarios pre-establecidos. Igualmente consideramos importante lograr un adecuado nivel de motivación, independencia e iniciativa.

5. En el área de ocupación vemos que un mínimo porcentaje de educandos poseen un adecuado manejo de las normas de seguridad e higiene en el desempeño da una tarea, lo que trae como consecuencia muchos riesgos por parte del alumno, es importante que esta área se refuerce con fines preventivos con la finalidad de no arriesgar su integridad personal, que si bien luego de la aplicación de nuestro programa se vienen dando pasos asertivos, se hace necesario profundizar estas acciones hasta llegar a un nivel óptimo.

6. Es pertinente que la estructura curricular contemple nociones de comercialización y venta, para que puedan colocar sus productos en el mercado y con ello percibir un valor monetario $y$ gratificaciones emocionales por el esfuerzo realizado.

\section{BIBLIOGRAFÍA}

Costallat, D. (1981) Psicomotricidad III. Buenos Aires: Losada.

Díaz, M (1980) Formación profesional y empleo del deficiente psíquico.

Madrid: Fuelabrada

Hans Willy Petzolt (1997). Técnica profesional. Ministerio de Educación. Senati (1988). Perfil

ocupacional.

Instituto Peruano de Seguridad Social (1997). Rehabilitación.

Thorburn,M.J .(1 983).Evaluación en la rehabilitación profesional de los deficientes mentales.

Organización Internacional del Trabajo: Ginebra.

Vasquez, F. (1995) El astronauta social. Gráfica y Asesores Creativos.

Whelan E.( 1983) El análisis de las tareas y las técnicas para formar a los deficientes mentales. Ginebra:

Organización Internacional del Trabajo 
ANEXOS

\section{PERFIL OCUPACIONAL COSMETOLOGÍA}

Tollex da cosmetrol.oGLA

\begin{tabular}{|c|c|c|c|c|}
\hline & Aspectos & & NIVELES & \\
\hline 1 & Retardo Mental & Moderado & & ve \\
\hline \multirow[t]{2}{*}{2} & Exactive Precisión & Oruesa & Media & \\
\hline & Pulso & Oruese & Medie & \\
\hline \multirow[t]{2}{*}{3} & Destrexa manual & Oruesa & & Find \\
\hline & Oestrexa digital & Oruesa & Media & ne \\
\hline 4 & $\begin{array}{l}\text { Armonia Coordinadón } \\
\text { Dimanual }\end{array}$ & Oruesa & Media & Pra \\
\hline 6. & Sociabilidad & Bajo & $R \in g u t a r$ & gupo \\
\hline o. & Desempeño ocupadonal & Balo & Medio & \\
\hline $\mathbf{7}$ & Atendón y Concentracesn & Balo & $R \in$ ould & no \\
\hline 8 & C bleulo & Bajo & & ueno \\
\hline o & Coordinación Vaomotax & Defidente & Reg & \\
\hline 10 & Disotimineas n Thoti & Defidente & & $\tan x$ \\
\hline 11 & Controt de Impulaon & Pobre & Fiegula & na \\
\hline 12 & Toleranda a ia Frustración & Pobre & $R=0$ & na \\
\hline 13 & Padencia & Pood & Reguiar & decluada \\
\hline 14 & $\begin{array}{l}\text { Disorimina lo tundamental de io } \\
\text { aooesorio }\end{array}$ & Defidente & Regular & ouse \\
\hline
\end{tabular}

Descripción:

Tiene que ver con el corte de cabello, ondulación, maquillaje, manicure, tratamiento capiral y tratamiento facial.

\section{Operaciones:}

Recorte de uñas, limado de uñas, remover cuticula, pintado de uñas. lavado de cabello, corte de cabello, cepillado de cabello. aplicación de base, colocación de líquidos, control de tiempo, secado de cabello, masaje al cuero cabelludo, aplicación de champú y reacondicionador, colocación de vaporizador, limpieza de cutis.

Conocimientos:

Conocimientos anatómicos básicos, clases de cabellos, tipos de cutis, Tipos de Champú y reacondicionador, tipo de limas, clases de tijeras, clases de corta-uñas. pinzas.

Características Psicológicas:

Retardo mental leve

- $\quad$ Buena atención y reflejos rápidos

Capacidad de diferenciar lo fundamental de lo accesorio

Capacidad para establecer buenas relaciones interpersonales

Paciencia

Buena tolerancia a la frustración

Exactitud: precisión fina y pulso fino.

Agilidad: destreza manual media y destreza digital fina

Armonía: uso de ambas manos y coordinación fina 


\section{PERFIL OCUPACIONAL: DECORACIÓN}

Twer de: DECORACION

\begin{tabular}{|c|c|c|c|c|}
\hline & ASPECTOS & & NIVELES & \\
\hline 1 & Retardo Mental & Moderado & & Leve \\
\hline \multirow[t]{2}{*}{2} & Exactiva Preasión & Gruesa & Pdia & Fina \\
\hline & Puiso & Gruesa & Mquia & Fina \\
\hline \multirow[t]{2}{*}{3} & Destreza manual & Oruesa & ia & Fina \\
\hline & Destreza digital & Gruesa & Media & Pra \\
\hline 4 & $\begin{array}{l}\text { Armonia Coordinación } \\
\text { Bimanual }\end{array}$ & Oruesa & dia & \\
\hline 5 & Sodabilidad & Bays & Pulat & Bueno \\
\hline o & Desempeño ooupadional & Bajo & Mado & Aito \\
\hline 7 & Atendón y Concentración & Bajo & at & Bueno \\
\hline 8 & C sioulo & $\theta$ ajo & ul at & Gueno \\
\hline 9. & Coordinacibn Visomotaz & Deticiente & $R \in \mathcal{P} u$ ar & Buena \\
\hline 10 & Disorimina dón Tact & Deticiente & Redulat & Buena \\
\hline 11 & Control de impulsos & Pobre & $R=$ quilar & Buena \\
\hline 12 & Toletanca a ta Frustración & Pobie & $R \in g u l a t$ & Bupena \\
\hline 13 & Padenca & Pooa & Requiar & Secuada \\
\hline 14 & $\begin{array}{l}\text { Disorimina lo Aundamental de lo } \\
\text { Acoesorio }\end{array}$ & Deficiente & $R \in g u l a r$ & Bueno \\
\hline
\end{tabular}

\section{Descripción:}

Comprende actividades de estampados con sellos y con plantillas en servilletas, paneras, manoplas, individuales y manteles, realiza estampados con plantillas, pinturas con pinceles, decorado de abanicos, decorado de retablos ayacuchanos..

Operaciones:

Estampar, delinear, pintar, matizar colores, acabados.

Conocimientos:

Tipo de pinturas para telas, sellos y plantillas, acabado con aguja e hilo, blondas y grecas, tipos y usos de los pinceles.

Características Psicológicas:

\section{Retardo mental leve}

- $\quad$ Agudeza visual

- $\quad$ Exactitud: precisión fina y pulso fino.

- $\quad$ Agilidad: destreza manual media y destreza digital fina

- Armonía: uso de ambas manos y coordinación fina

- Paciencia

- Nivel de sociabilidad : promedio

- $\quad$ Nivel de desempeño ocupacional : promedio 


\section{PERFIL OCUPACIONAL: BIO - HUERTO}

Taller do BIO - HUERTO

\begin{tabular}{|c|c|c|c|c|}
\hline & ASPECTOS & & NIVELES & \\
\hline 1 & Retatde Mental & Moderado & & Leve \\
\hline \multirow[t]{2}{*}{2} & Exactitud Precasion & G5es. & Media & Fina \\
\hline & Pulso & oresa & Medis & Find \\
\hline \multirow[t]{2}{*}{3} & Destreza manual & artera & Media & Fina \\
\hline & Destreza digital & ortesa & Media & Fina \\
\hline 4 & $\begin{array}{l}\text { Armonia Coordinadón } \\
\text { Birnanual }\end{array}$ & $\mathbb{1}_{0.4}$ & Medila & Find \\
\hline 6. & Socabilidad & $\theta$ ajo & Repular & Bueno \\
\hline 의 & Desemp eǨo o cupadonal & B alo & Mofio & Alto \\
\hline 7 & Atención y Concentración & $\theta$ ajo & Recutar & Bueno \\
\hline 8 & C bloulo & $\theta$ anjo & gular & Bueno \\
\hline 이 & Coordinación Vsomotix & Dendente & Poular & Buena \\
\hline 10 & Diserimina dón Thetil & Dendent- & Regular & Buena \\
\hline 11 & Control de Impulsos & Pobre & R.Pular & Buena \\
\hline 12 & Toleranda a Ia Fiustración & Pobre & Refuar & Buena \\
\hline 13 & Padenda & Pood & Refuar & Adeasada \\
\hline 14 & $\begin{array}{l}\text { Diserimina to tundamental de io } \\
\text { acoesono }\end{array}$ & Oefidente & Reoular & Bueno \\
\hline
\end{tabular}

Descripción:

Comprende la preparación del terreno de cultivo, abonado, siembra, regado y podado de plantas uy hortalizas, hidroponía.

\section{Operaciones:}

Remoción de tierra, rastrillar, hacer surcos, sembrar, abonar, regar, podar, cosechar, sembrar almácigos.

Conocimientos:

Manejo de herramientas agrarias básicas, pala, lampa, pico, rastrillo, tijera, cortadora de césped, espátula, manguera, carretilla. Diferenciar diversos tipos de plantas y malezas, conocer los periodos, de riego.

\section{Características Psicológicas:}

Retardo mental moderado

Coordinación manual

Exactitud: precisión gruesa, pulso grueso.

Agilidad: destreza manual gruesa, destreza digital gruesa.

Armonía: uso de ambas manos y coordinación gruesa

Paciencia

Capacidad para seguir instrucciones. 


\section{PERFIL OCUPACIONAL: CERAMICA BÁSICA}

Toller 4h: CBRABuCA BASICA

\begin{tabular}{|c|c|c|c|c|}
\hline & ASPECTOS & & HIVELES & \\
\hline 1. & Retardo Mental & Moderado & & Leve \\
\hline \multirow[t]{2}{*}{2} & \multirow[t]{2}{*}{ Predisión } & orpess & Media & Fina \\
\hline & & oifesa & Media & Fina \\
\hline \multirow[t]{2}{*}{3} & \multirow{2}{*}{$\begin{array}{l}\text { Destieza manual } \\
\text { Destieza dighal }\end{array}$} & Gruesa & Media & Fina \\
\hline & & Gruesa & Media & Fins \\
\hline 4 & $\begin{aligned} \text { Armonia Coordinación } & \\
& \text { Bimanual }\end{aligned}$ & crues & Media & Fins \\
\hline 6) & Sodabilidad & B ajo & Regular & Bueno \\
\hline 요 & Desempeño ooup a donal & Bajo & Medio & Alto \\
\hline 7 & Atención y Concentradín & Bajo & Regular & Bueno \\
\hline \multicolumn{2}{|r|}{8 Caloulo } & Bajo & Regular & Bueno \\
\hline ○) & Coordinación Visomotiz & Defidiente & Repular & Buena \\
\hline 10 & Disotimin a dón T́́cti & Deficiente & Regular & Buena \\
\hline 11 & Contrel de impuisos & Pobre & Refoulat & Buend \\
\hline 12 & Tolerancia a la Frustraoión & Pobre & Regulat & Ouena \\
\hline 13 & 3 Padenda & Poca & Refoluat & Adecuada \\
\hline 14 & $\begin{array}{l}4 \text { Disorimina lo tundamental de to } \\
\text { Aocesorio }\end{array}$ & Defidente & Regulat & Bueno \\
\hline
\end{tabular}

Descripción:

Realiza trabajo de modelado en arcilla, horneado de cerámica, dibujo, pintado y acabado.

Operaciones:

Amasar, moldear, rascar, dibujar, pintar.

Características Psicológicas:

Retardo mental moderado

- Capacidad para trabajar en equipo, capacidad para seguir instrucciones, desarrollo de buenos hábitos de trabajo, movimiento simultáneo de ambas manos para evitar el cansancio y favorecer la rapidez. Movimientos digitales, Precisión gruesa, agudeza visual.

- $\quad$ Agilidad: destreza manual gruesa.

- Armonía: uso de ambas manos y coordinación gruesa

Nivel de sociabilidad: promedio

- $\quad$ Nivel de desempeño ocupacional: medio bajo. 


\section{PERFIL OCUPACIONAL: CARPINTERÍA}

\begin{tabular}{|c|c|c|c|c|}
\hline & ASPECTOS & & NIVELES & \\
\hline 1 & Retardo Mental & Moderado & & Leve \\
\hline \multirow[t]{2}{*}{2} & Exactived Preasión & Gruesa & & Fina \\
\hline & Pulso & Gruesa & & Fina \\
\hline \multirow[t]{2}{*}{3} & Destreza manual & Gruexa & & Fine \\
\hline & Destrexa digital & Qruesa & dia & na \\
\hline 4 & $\begin{array}{r}\text { Armonia Coordinación } \\
\text { Bimanual }\end{array}$ & Oruesa & & rine \\
\hline$\sigma$ & So dabilidad & B ajo & Requin & Bueno \\
\hline o & Desempefio o cupadiona! & B ajo & Medio & \\
\hline 7 & Atención y Concentradón & Bajo & $R \bullet$ & Bưno \\
\hline$\theta$ & C biculo & Oajo & & eno \\
\hline 임 & Coordinadón Vsomotix & Defidiente & Re & ins. \\
\hline 10 & Disoriminación Thoti & Defidiente & Refular & Buena \\
\hline 11 & Control de Impulsos & Pobre & Re्णित्र & Buena \\
\hline 12 & Toletanda a la Frustracib́n & Pobre & Re oular & Pna \\
\hline 13 & Padencia & Pood & Requalar & Aderuad a \\
\hline 14 & $\begin{array}{l}\text { Diserimina lo Nundamental de lo } \\
\text { aooesorio }\end{array}$ & Defielente & Regular & even. \\
\hline
\end{tabular}

Descripción:

Confecciona muebles de madera, de calidad según planos utilizando maquinas y herramientas de carpintería apropiadas.

Ejecuta el trazado sobre la madera, para el aserrados, realizar cortes y entalles. Hace los ensambles necesarios, utilizando herramientas para el montaje de muebles, arma las partes ensamblándolas y fijándolas.

Coloca tiradores en los muebles según estilos. Pinta, charola.

Tareas Típicas:

Construcción de palitos de tejer, tablas de picar, servilletero;, papeleras, azafatas. repisas, portallaves, mesitas de centro, zapateras, posa ollas, posa pirex.

Conocimientos:

Maderas: tipos, accesorios de fijación, materiales adhesivos, materiales de acabado. Herramientas de uso manual, maquinas y equipos. Seguridad y limpieza.

Maquinas:

Sierra cintas, sierra radial, gargola, cepilladora, torno simple, taladro, esmeril.

Herramientas:

Serrucho, martillo, cepillo, birbiquí, tenaza, metro, formones. destornillador, cuchilla de vuelta. alicate, compás, mazos, prensas, limas, brocas, llave de boca, piedra de asentar. 


\section{Materiales:}

Maderas, cola, clavos, tornillos. bisagras. tiradores. perillas, reten magnético. lijas. terokal. gomalaca, alcohol, lacas. thiner. aguarras. ceras. pitas, tizas. pinturas.

\section{Características Psicológicas:}

- $\quad$ Retardo mental leve

- $\quad$ Percepción de formas y volúmenes

- $\quad$ Atención y Concentración

- $\quad$ Coordinación viso motriz

- $\quad$ Movilidad y fuerza en brazos y piernas

- Coordinación bimanual

- Resistencia de la bipedestación

- Percepción Táctil

- Percepción visoespacial

- $\quad$ Exactitud: precisión media y pulso fino

- $\quad$ Agilidad: destreza manual media y destreza digital fina

- Armonía: uso de ambas manos y coordinación media.

- Paciencia.

- $\quad$ Nivel de sociabilidad: media

- $\quad$ Nivel de desempeño ocupacional: Alto. 